KARL JANSEN-WINKELN

\title{
Eine Bau- und Bittinschrift am Tempel von Luxor
}

\author{
Hierzu Tafel I-VIII
}

Auf dem nordöstlichen Teil der Außenwand des Vorhofes Amenophis' III. im Luxortempel, rechts des Durchgangs, findet man etwa in Augenhöhe eine Inschrift von 20 Kolumnen aus dem Jahr 4 (2. prt) von Philipp Arrhidaios ${ }^{2}$, verfasst von einem Amunpriester, königlichen Schreiber und Bauleiter ( $h r p k 3 t$ ) namens ${ }^{c} n h-p$; hrd, Sohn eines Amunpriesters und königlichen Schreibers Jrt-Hr-r.w und einer T3-hij-bj3t. Diese selben Leute sind auch von dem Würfelhocker Kairo JE 37989 (aus der Cachette von Karnak) her bekannt, den ${ }^{c} n h$ - $p$; - hr $r d$ seinem Vater Jrt-Hr$r . w$ gestiftet hat ${ }^{3}$.

Die Inschrift am Luxortempel ist 1893 von G. Daressy veröffentlicht worden ${ }^{4}$, in Drucktypen (nach links blickend) und ohne weiteren Kommentar. Seine Wiedergabe des Textes ist unvollständig und enthält zahlreiche Fehler, auch an Stellen, die für das Verständnis entscheidend sind. Selbst die allgemeine Gliederung der Inschrift ist daher anhand dieser Abschrift nicht zu entschlüsseln. 90 Jahre später ist die Inschrift neu publiziert und auch übersetzt wor$\operatorname{den}^{5}$. Diese neuerliche Wiedergabe des Textes ist aber nichts anderes als eine exakte Kopie der Abschrift Daressys, mit allen Fehlern und Aus-

\section{PM II², 335 (219b), s. Plan XXXI (219).}

Entsprechend April/Mai 320 v. Chr.

$\mathrm{K}$. Jansen-Winkeln, Biographische und religiöse Inschriften der Spätzeit, ÄUAT 45, 2001, 179-184; 408-409; Taf. 63-64. Auf die Identität dieser Personen hat zuerst $H$. De Meulenaere hingewiesen, in: S. Vleeming (ed.), Hundred-gated Thebes, P.L. Bat. 27, 1995, 88; id., in F. Tiradritti, Die Schatzkammer Ägyptens, München 2000, 340-341.

RecTrav 14, 1893, 33 (LIV).

M. Abder-Raziq, "Ein Graffito der Zeit Alexanders des Großen im Luxortempel", in: ASAE 69, 1983, 211-215. lassungen; die Übersetzung ist dementsprechend wenig hilfreich. In jüngster Zeit hat G. Gorre diejenigen Abschnitte neu behandelt, die sich auf die Bautätigkeit des ${ }^{c} n h-p 3-\underline{h} r d$ beziehen ${ }^{6}$. Da er sich aber weitestgehend auf die Arbeiten von Daressy bzw. Abd er-Raziq stützt, ist das Ergebnis gleichfalls unbefriedigend. Auch aus seiner Bearbeitung geht nicht hervor, welche Ereignisse überhaupt berichtet werden.

Sonst wird diese Inschrift nur recht selten erwähnt ${ }^{7}$, und das ist eigentlich erstaunlich: Sie ist nicht nur bequem zugänglich und fast vollständig erhalten, sondern auch von ungewöhnlichem Interesse, denn sie berichtet von zwei Tempelerweiterungen, mit genauen Angaben der Ausmaße des Bauwerks und der Bauzeit mehr als ungewöhnlich in einer Privatinschrift. Der Grund für die mangelnde Beachtung ist offensichtlich, dass dieser Text verwittert, schwer lesbar und verständlich ist, was schon Daressys Schwierigkeiten bei der Abschrift erklärt. Ich selbst habe die Inschrift mehrfach im

${ }^{6}$ G. Gorre, Les relations du clergé égyptien et des Lagides d'après les sources privées, Studia Hellenistica 45, Löwen 2009, 53-57.

J. A. Janssen, OMRO 31, 1950, 33 (Foto); 36; 38 (24) (Ausschnitt aus Z. 19 bzgl. der 110 Jahre Lebenszeit); W. Peremans u. a., Prosopographia Ptolemaica, III, Löwen 1956, 115 (Nr. 5861); Jansen-Winkeln, ZÄS 132, 2005, 35, n. 1; B. McClaine, in: P. Dorman B. Bryan (edd.), Sacred Space and Sacred Function in Ancient Thebes, SAOC 61, 2007, 90; G. Vittmann, in: M. Hasitzka et al. (edd.), Das Alte Ägypten und seine Nachbarn. Festschrift zum 65. Geburtstag von Helmut Satzinger, Krems 2003, 170, n. 43; D. Schäfer, „Alexander der Große. Pharao und Priester", in: St. Pfeiffer (ed.), Ägypten unter fremden Herrschern zwischen persischer Satrapie und römischer Provinz, Frankfurt a. M. 2007, 59 (,eine Art Bauinschrift für das Alexandersanktuar"). 
Original studiert ${ }^{8}$, ohne aber alles vollständig und richtig lesen zu können. Die hier vorgelegte Abschrift und Bearbeitung' (s. Taf. I-V) enthält daher auch noch einige Lücken und zahlreiche Unsicherheiten; es ist nur ein erster Versuch.

Wichtig ist aber, dass die Struktur des Textes deutlich wird, seine interne Gliederung und die unterschiedlichen Textsorten, aus denen er besteht. Insgesamt gibt es drei größere Abschnitte mit insgesamt elf Paragraphen:

A) Einleitung: Datum, Sprecher und Adressaten: Z. $1-5$
a) Datum: Z. 1 (Jahr 4 Philipp Arrhidaios)
b) Sprecher: Z. 1-3
c) Adressaten: Z. 3-5 $5^{10}$

B) Biographische Bauinschrift: Z. 5-10

d) Bau eines „Goldhauses“ im Tempel von Luxor im Jahr 1 Alexanders des Großen: Z. 5-7

e) Beförderung zum Baumeister im Jahr 3 des Philipp Arrhidaios: Z. 7-8

f) Bau eines Goldhauses im Tempel von Karnak im selben Jahr: Z. 8-10

C) Bitten um Belohnung durch Gott und Nachwelt:

Z. $10-20$

g) Anruf an Amun mit Eulogie: Z. 10-12

h) Vorstellung Beter und Gebet: Z. 12-14

i) Vorstellung Sohn und Fürbitte für ihn: Z. 14 16

j) Anruf an die Lebenden und Bitte für den Sprecher und seinen Sohn: Z. 16-18

k) Sicherungsformel für den Sprecher und seinen Sohn: Z. 18-20

\section{Übersetzung $^{11}$}

a) Datum

\section{1 rnpt-zp 4, 2. prt hrr ḥm (n) nswt-bjt Prpys}

$1 \mathrm{Jahr} 4^{(1)}$, zweiter Monat der prt-Jahreszeit unter der Majestät des Königs von Ober- und Unterägypten Philippos:

${ }^{8}$ Außerdem an Fotos, u. a. solchen, die S. Grallert und A. Klug freundlicherweise für mich angefertigt hatten.

Die „Übersetzung“ in ASAE 69, 211-215 kann man kaum so nennen; ich werde im Folgenden nicht weiter darauf eingehen.

${ }^{10}$ In Form eines „Anrufs an die Lebenden“, aber obne die übliche anschließende Bitte. Sinngemäß könnte man ergänzen: „hört, was ich zu sagen habe“.

${ }^{11}$ Ich danke G. Vittmann für einige sehr nützliche Hinweise. b) Sprecher bzw. Autor

hm-ntr Jmn m Jpt-swt zm3itj n K3-mwt.f hntj W3st rh nswt žs (nswt) 2 žs Jmn jw.f jp n p3 4 z 3w hrp k3t

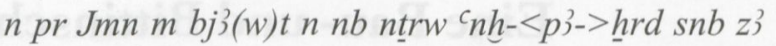
$n$ him-nț Jmn m Jpt-swt rh 3 nswt žs (nswt) Jrt-Hrr.w mj $^{\mathrm{c}}$-hrw hr Wsjr jr(t).n nbt-pr jhyt $n$ Jmn-rn.f T)-hjj-bj3t m3c-hrw 3wt-jb hr Zk(r) dd.f

Der Prophet des Amun in Karnak, der $z m^{3} t j$ Priester des Kamutef, des Ersten von Theben, der Königsbekannte und (königliche) Schreiber, 2 der Rechnungsschreiber des Amun ${ }^{(2)}$ der vier Phylen, der Leiter der Arbeit(en) des Hauses des Amun an den Wunderwerken für den Herrn der Götter ${ }^{c} n h-\left(p^{3}-\right) h r d^{(3)}$, der wohlbehalten ist, der Sohn des Propheten des Amun in Karnak, des Bekannten 3 des Königs und (königlichen) Schreibers Jrt-Hr-r.w, gerechtfertigt bei Osiris, geboren von der Hausherrin und Musikerin dessen ,Der seinen Namen verbirgt ${ }^{6}$ T3-hjj-bj3t ${ }^{(4)}$, gerechtfertigt, fröhlich bei Sok $(\operatorname{ar})^{(5)}$, indem er sagt:

c) Adressaten: Anruf an die Leser der Inschrift

$j$ hmww-ntr $n b 4 w^{c} b \quad n b \underline{h} n w-z 3 \quad n b{ }^{c} q r$ pr pn sir $n b$ $n w$ pr-Jmn žs $n b \check{s} s(3) m z \check{s}(w){ }^{c} d-m r n b m$ 3bd.sn $n t j(j w) . s n(?)(r) \check{s} d j z \check{s}$ pn hr $\mathbf{5} \underline{d} d$

„O alle Propheten ${ }^{(6)}, 4$ alle Wab-Priester, alle Gelehrten (o. ä.) ${ }^{(7)}$, die eintreten in dieses Haus, alle Gebildeten(?) ${ }^{(8)}$ des Hauses des Amun, alle Schreiber, die erfahren sind ${ }^{(9)}$ in den Schriften, alle ${ }^{c} d-m r$-Priester ${ }^{(10)}$ in ihrem Monat, die diese (In)Schrift lesen (werden) durch ${ }^{(11)} \mathbf{5}$ das Sprechen (folgendermaßen):

\section{d) Bau des Goldhauses im Luxortempel}

rnpt-zp 1, 1. 3ht, sw $1 \mathrm{hr} h \mathrm{hm}$ (n) nswt-bjt 3rksndrjs

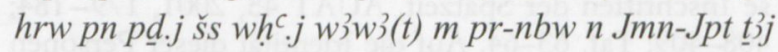
ntrw 6 ntr c $3 c_{n h}$ hrj ntrw $m$ jnr hạ nfr rwd q3w.f

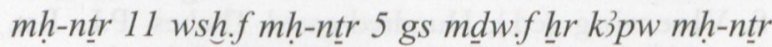
9 grh $n$ wìh 3 t nfry $(t)$ (r) 7 2. 3ht sw 9 m rnpt tn $\mathrm{mh}$ hrw 39

Jahr 1, erster Monat der 3ht-Jahreszeit, Tag 1 unter der Majestät des Königs von Ober- und 
Unterägypten Alexandros ${ }^{(12)}$ : an diesem $\mathrm{Tag}^{(13)}$ habe ich den (Mess-)Strick ausgespannt und die Schnur (wieder) gelöst ${ }^{(14)}$ im Goldhaus des Amenope, des männlichsten der Götter ${ }^{(15)}, 6$ des groBen lebenden Gottes, des Obersten der Götter, (das gebaut ist) aus schönem hellem Sandstein ${ }^{(10)}$; seine ,Höhe ${ }^{6}$ (= Länge) ist 11 Gottesellen ${ }^{(17)}$, seine Breite 5(?) 1/2 Gottesellen ${ }^{(18)}$, seine Tiefe unter dem Dach(?) ${ }^{(19)} 9$ Gottesellen. Der Abschluss des (Ver-)Legens des Hartsteins(?) (erfolgte) bis (zum) 7 2. Monat des 3 h ${ }^{(20)}$ Jahreszeit, Tag $9(?)^{(21)}$ in diesem Jahr, was 39 Tage ergibt ${ }^{(22)}$.

e) Beförderung zum Baumeister

rnpt-zp 3, 3. 3ht, sw $1 \mathrm{hr} h \mathrm{hm}$ (n) nswt-bjt Prpys hrw pn whm nb ntrw 8 hzwt rdj.n.f wj $m$ hrp kit $m$ pr.f $m b j 3(w) t . f$

Jahr 3, 3. Monat der 3ht-Jahreszeit, Tag 1(?) unter der Majestät des Königs von Ober- und Unterägypten Philippos: an diesem Tag wiederholte(?) ${ }^{(24)}$ der Herr der Götter $\mathbf{8}$ die Gunst und er setzte mich ${ }^{(25)}$ ein als Leiter der Arbeit in seinem Haus an seinen Wunderwerken ${ }^{(26)}$.

\section{f) Bau des Goldhauses im Karnaktempel}

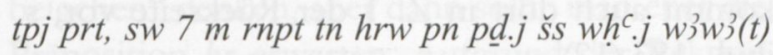
9 m pr-nbw $n$ Jmn-R $R^{c}$ nswt-ntrw m jnr nfr rwd q3w.f mh-ntr $14 r^{3} 4$ hn(w) jwnyt wsh.f mh-nț 14 (?) hnw n jwnyt mdw.f hr kjpw mh-ntr $9 r^{\prime} 310$ grh hrc 3 t nfry $(t r)$ 4. šmw sw 1 [6] m rnpt tn $r 3 b d 7 r^{3} 3$

1. Monat der prt-Jahreszeit, Tag 7 in diesem Jahr: an diesem Tag habe ich den (Mess-)Strick ausgespannt und die Schnur (wieder) gelöst 9 im Goldhaus des Amonrasonther (das gebaut ist) ${ }^{(27)}$ in schönem Sandstein; seine "Höhe' (= Länge) ist 14 Gottesellen ${ }^{(28)}$ : der 4. Teil(??) des Inneren ${ }^{(29)}$ der Pfeilerhalle(?) ${ }^{(30)}$, seine Breite ist $[1] 4(?)^{(31)}$ Gottesellen: der Ruheplatz (?) der Pfeilerhalle(? $)^{(32)}$, seine Tiefe unter dem $\operatorname{Dach}(?)^{(33)}$ ist 9 Gottesellen [und $1 / 3$ ??] $]^{(34)} \cdot 10$ Der Abschluss mit dem Hartstein(?) ${ }^{(35)}$ (währte) bis (zum) 4. Monat der šmw-Jahreszeit, Tag 1[6] ${ }^{(36)}$ in diesem Jahr, entsprechend 7 Monate und 1/3.

\section{g) Anruf an Gott und Eulogie}

$c_{n h-p}$-hind dd.f $j$ Jmn-R ${ }^{c}$ nswt-ntrw jmn-rn.f $11 \mathrm{jrj}$

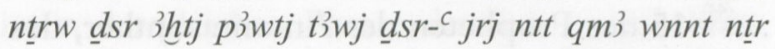
špsj shạ t3wj ptr t3 mj qd.f b3 wr n 12 ntrw ntr ${ }^{3}$ n

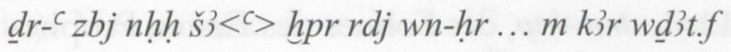

$c_{n h-p}$ - $-\underline{h} r d$, indem er sagt: „O Amonrasonther, ,Der seinen Namen verbirgt', 11 der die Götter gemacht hat, dessen beide "Horizonte abgeschieden sind, Urzeitlicher der Beiden Länder, mit heiligem Arm, der gemacht hat, was ist, und geschaffen hat, was existiert, erhabener Gott, der die Beiden Länder erleuchtet, den das ganze Land erblickt ${ }^{(37)}$, großer Widder 12 der Götter, großer Gott des Anbeginns, der die Ewigkeit durchlebt und das Werden begonnen hat, der bewirkt die Gesichtsöffnung ${ }^{(38)}$... im Schrein seines Udjat-Auges ${ }^{(39)}$ :

\section{h) Vorstellung Beter und Gebet für sich}

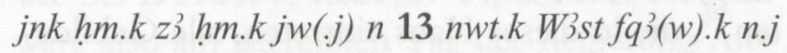

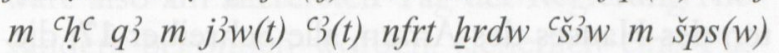

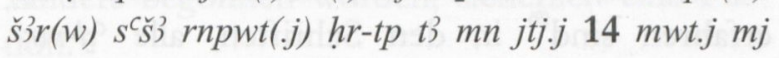
jrt.n.j

Ich bin dein Diener, der Sohn eines Dieners von $\operatorname{dir}^{(40)}$, indem (ich) $13 \mathrm{zu}$ deiner Stadt Theben gehöre $^{(41)}$. Deine Belohnung für mich bestehe in einer hohen Lebenszeit, einem langen schönen Alter, zahlreichen Kindern, die angesehen und gebildet sind, dem Vermehren(??) ${ }^{(42)}$ (meiner) Jahre auf Erden, dass (am Leben) bleiben mein Vater 14 und meine Mutter, entsprechend dem, was ich getan habe.

\section{i) Vorstellung Sohn und Fürbitte für ihn}

z3.j wr m špsj s3r Jrt-Hr-r.w snb jr(t).n nbt-pr ihyt Jmn-rn.f ${ }^{c} n h-t 3-h s^{3} t$ [z $\left.3 t n\right] m r \ldots 15$ hm-ntr Jmn-R ${ }^{c}$

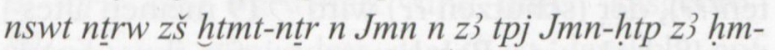
ntr tpj Ns-p3wtj-t3wj mj jrj.n.f r jtj.f wr zp 2 Hr-z3$3 s t \ldots f q 3 w 16$ n.f $m$ rnpwt $96 \underline{h r d w} 10[+\mathrm{x}] m \check{s} p s j$ $s 3 r$

(Mein) ältester Sohn ist ein Angesehener und Gebildeter (namens) Jrt-Hr-r.w, der wohlbehal- 
ten ist, geboren von der Hausherrin und Musikerin dessen, ,Der seinen Namen verbirgt $c_{n h(?)-t 3-h s^{3} t}(? ?)^{(43)}$, der Tochter des Vorstehers ... ${ }^{(4)} 15$ des Propheten des Amonrasonther, des Schreibers des Gottesschatzes des Amun in der 1. Phyle Jmn-htp, des Sohnes des. Ersten Propheten $N s-p 3 w t j-t 3 w j^{(45)}$, so wie er gehandelt hat entsprechend seinem Vater ${ }^{(46)}$.

Wie groß ist Harsiese (?) ... ${ }^{(47}$

Die Belohnung 16 für ihn bestehe in (weiteren) 96 Jahren, $10[+\mathrm{x}]^{(48)}$ Kindern, die angesehen und gebildet sind.

\section{j) Anruf an die Lebenden mit Fürbitte für Sohn ${ }^{(49)}$}

$j h m w-n t r n b w^{c} b \quad n b \underline{h} n w-z 3 n b^{c} q r p r p n s i r w n b n w$

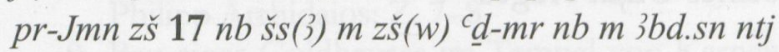
(jw).sn(?) (r) šdj zš pn sh̉̉.tj.fj rn.j (r) tp-nfr .. 18

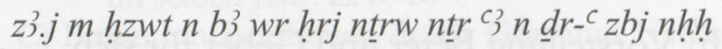

O alle Propheten, alle Wab-Priester, alle Gelehr$\operatorname{ten}^{(50)}$, die in dieses Haus eintreten, alle Gebildeten des Hauses des Amun, alle Schreiber 17, die erfahren $\operatorname{sind}^{(9)}$ in den Schriften, alle ${ }^{c} d-m r-$ Priester in ihrem Monat, die diese (In)Schrift lesen, die meines Namens gedenken werden (in) guter Weise(?) $)^{(51)} \ldots 18$ meinen Sohn in der Gunst des großen Widders, des obersten der Götter, des großen Gottes des Anbeginns, der die Ewigkeit durchlebt.

\section{k) Sicherungsformel}

$j r w^{c} b(w) n b$ hmm(w)-nțr (?) [nd.tj(??)].fj [z3] $19 . j$ wr fq3(w) n.f (n) nb ntrw m rnpt 110 hrdw ntj $m$ špsj sirw qrs.tw.f ... 20 r-gs jtj.f mwt.f mj jrj n.f z3.f wr $m$ špsj sir jwjw dt $n h h \underline{h}$

Was betrifft jeden Wab-Priester(?), Propheten(??), der (schützen ??) wird ${ }^{(52)} 19$ meinen ältesten [Sohn], eine Belohnung sei für ihn $(\mathrm{des})^{(53)}$ Herrn der Götter bestehend aus 110 (Lebens-) Jahren und Kindern, die angesehen und gebildet sind, er möge begraben werden ... ${ }^{(54)} 20$ an der Seite seines Vaters und seiner Mutter, entsprechend dem, was (seinerseits) sein ältester
Sohn für ihn tut ${ }^{(55)}$ als ein Angesehener und Gebildeter und die, die in alle Ewigkeit kommen werden.

\section{Anmerkungen}

(1) Der Strich links ist beschädigt, aber nach den Spuren und der räumlichen Verteilung der Striche ist zweifellos (Jahr) 4 zu lesen.

(2) Vgl. zu diesem Titel Traunecker, in: W. Clarysse et al. (edd.), Egyptian Religion: the Last Thousand Years, Studies Dedicated to the Memory of Jan Quaegebeur, II, OLA 85, 1998, 1205-1206 (f); G. Vittmann, Der demotische Papyrus Rylands 9, II, ÄUAT 38, 1998, 548.

(3) In Z. 10 wird der Name mit $\square$ unter dem sitzenden Kind geschrieben. Das wäre auch hier zu erwarten, und Daressy (RecTrav 14, 33) hat es auch so gelesen, aber ich kann von diesem $p$ keine Spur entdecken.

Derselbe ${ }^{c} n h-p 3-\underline{h r d}$ hat seinem Vater $\mathrm{Jrt}-\mathrm{Hr}$ r.w (hier in Z. 3 genannt) den Würfelhocker Kairo JE 37989 gestiftet, s. Jansen-Winkeln, Biographische und religiöse Inschriften, 179184; 408-409; Taf. 63-64 (Nr. 29). Zu seinen Titeln zm3tj $n$ K3-mwt.f rh njswt žs njswt ž́ Jmn jw.f jp n p3 jfdw z3w ibid., 182, Anm. 1-2. Die Haplographie rh njswt žs (njswt), hier in Z. 1-2, kommt auch dort in Z. 1 der Rückseite vor, s. ibid., 183 (12).

(4) Zum Namen s. die Angaben ibid., 183 (13).

5) Auf der Statue JE 37989 wird der Vater des Besitzers (und Großvater des $c_{n h-p}$ j-hrrd) namens $N s-J n j-h r t$ als $m{ }^{3}$-hrrw $3 w t-j b$ hr Zkr bezeichnet, s. Jansen-Winkeln, Biographische und religiöse Inschriften, 408, 182-183, Anm. 3. Es handelt sich also um ein Beiwort für einen Toten, und es dürfte sich wohl auf die Teilnahme am Sokarfest beziehen, die sich der Tote wünscht.

(6) Wörtlich der gleiche Anruf an die Lebenden (mit lediglich orthographischen Varianten) in Z. 16-17.

(7) Zur Bezeichnung $\underline{h} n-z\}$ s. zuletzt L. Morenz, GM 167, 1998, 5 („Kundige des sakralen Wissens"). 
(8) Das merkwürdige Inschrift nicht weniger als sechsmal vor, in Z. 16 in der gleichen Phrase wie hier, in den anderen vier Fällen jeweils in der Verbindung špsj(w) पे । (Z. 13; 14; 19; 20), in Z. 13 und 19 zusätzlich durch 迹 determiniert. Es ist jedenfalls eine Bezeichnung, mit der Personen positiv charakterisiert werden, etwas Erwünschtes: In Z. 14 wird der älteste Sohn des Sprechers damit beschrieben, in Z. 13, 19 und 20 ist es eine Eigenschaft, die seine Nachkommen haben sollen. Die Determinierung mit 迹 sowie die Stellung zwischen $h n-z)$ („Gelehrte, Theologen“ o. ̈..) und $z \check{s} w$ (,Schreiber") hier in Z. 4 spricht dafür, dass es sich um Leute mit intellektueller Fähigkeit handelt. Es sollte daher am ehesten eine ungewöhnliche Form von $s 3 r$, „klug, weise“ sein, aber die ständige Schreibung mit $\mid$, die sonst bei dieser Wurzel nicht vorkommt, ist vielleicht von Bedeutung: In Z. 4 und 16 sind damit die Leute bezeichnet, die diese Inschrift lesen bzw. ,sprechen" (Z. 5) (können), in den anderen Fällen sollen die (herangewachsenen) Nachkommen „angesehen“ und s3r sein. Der Ausdruck bezeichnet also in dieser Inschrift möglicherweise spezifisch den (in der alten Sprache) „Lesekundigen" und wurde daher geschrieben, als ob es mit „Mund“ zu tun habe.

(9) Man würde hier (und ebenso in Z. 17) aufgrund des Determinativs zunächst an $z n j$,vorbeigehen" denken, aber dann sollte man eher die Präposition $h r$ erwarten. Aufgrund der Rektion mit $m$ sowie des Kontextes ist wohl eher $s ̌(\beta)$ zu lesen $(z \check{s} w \quad n b \quad z \check{s}\} \quad m z \check{s}$, alle Schreiber, die in der Schrift geübt sind"). Immerhin wäre auch $z n j$ durchaus nicht unpassend, vielleicht hat der Steinmetz das Determinativ aufgrund einer solchen Assoziation gesetzt.

(10) Zu den ${ }^{c} d$ - $m r$-Priestern s. Wb I, 240,12; Daumas, MDAIK 16, 1958, 78, n. 9; Sauneron, BIFAO 63, 1965, 82-83 (cc); R. Parker u.a., The Edifice of Taharqa, BES 8, 1979, 59, n. 61.

(11) Die Bedeutung der Phrase ist klar, aber ihr Verständnis dennoch problematisch. Ich hatte sie zunächst als $n t j(\underline{\underline{\underline{\omega}}}) m \check{s} d j$, die Lesende sind“ $=$,die lesen“" verstanden. Die Schreibung von $n t j$ mit der Buchrolle ist selten, aber belegt, s. Wb II, 351 (unten); Jansen-Winkeln, Spät- mittelägyptische Grammatik, ÄUAT 34, 1996, \$243. Das Zeichen unter könnte man hier (mit Daressy) zur Not als « lesen, aber in Z. 17 ist in der gleichen Phrase deutlich - $\rightarrow$ geschrieben (was auch in Z. 4 die bessere Möglichkeit ist). Man müsste dann in Z. $17 \mathrm{zu} n t j$ emendieren, sicher nicht sehr naheliegend. G. Vittmann hat daher vorgeschlagen, beide Male $-\rightarrow$ zu lesen und die ganze Phrase als $n t j$ (jw.)sn (r) $\check{s} d j$ „die lesen werden“ $z u$ verstehen, also einen Relativausdruck des „Jüngeren Ägyptisch“. Eine derartige Formulierung (bei der man zudem $j w$ ergänzen müsste) wäre $\mathrm{m}$. W. aber in den „Anrufen an die Lebenden" ohne jede Parallele. Beide Möglichkeiten sind nicht recht befriedigend.

Das $h r$ (und vor allem das $r$ ) am Ende der Kolumne ist etwas unförmig, aber wohl sicher (ähnlich in Z. 5). Die Formulierung mit $h r+$ Infinitiv ist grammatisch unproblematisch, phraseologisch aber ungewöhnlich.

(12) Es ist ohne Zweifel 嚕 zu lesen, nicht wie bei Daressy, RecTrav 14, 33. Der Neubau wäre also am allerersten Tag der Regierung Alexanders begonnen worden, sicherlich eine Fiktion.

(13) Trotz der etwas unförmigen Gestaltung der Zeichen ist sicherlich ${ }_{m}^{\square \odot}$ hrw pn gemeint. Die gleiche Sequenz (ebenfalls mit folgendem $p$ d.j.j šs $\left.w h^{c} . j w 3 w^{3}(t)\right)$ findet sich in Z. 8-9; dort (Z. 8) ist deutlich 미을 zu lesen, ebenso in Z. 7 (unter der Kartusche).

(14) Zu pd.j šs whe ${ }^{c} . j$ w3w3t s. Wb I, 567,12; I, 250,2; P. Wilson, A Ptolemaic Lexikon, OLA 78, 1997, 383; 193-194. Die Lesung $w h^{c}$ (hier und in Z. 8) ist sicher, Daressy hat das Zeichen in merkwürdiger Weise missverstanden. Die ganze Phrase wird hier wohl weniger die Gründungszeremonie als einfach den Beginn des Baus bezeichnen.

${ }^{(15)}$ Es ist ziemlich deutlich Jmn-jpt zu lesen, nicht (wie bei Daressy) Jmn- $R^{c}$. Darunter ist das tijj ntrw sehr verwittert (und unten ausgebrochen), aber zumindest das $t$ ? ist deutlich; auf keinen Fall kann man njswt ntrw lesen.

${ }^{(16)}$ Hier (ebenso in Z. 9) formuliert der Autor entweder sehr verkürzt, und es ist vor $m j n r$ so etwas wie „das nun gebaut ist" zu ergänzen bzw.

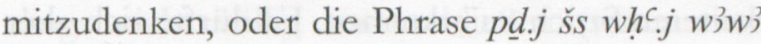


steht selbst schon für etwas wie ,ich errichtete, ich baute“.

(17) Der Einer-Strich steht eigentlich etwas zu tief, aber er sieht nicht so aus, als handele es sich nur um ein Loch im Stein. Daressy liest gleichfalls 11 .

Die „Gotteselle“ als Maß ist sonst nur in demotischen Texten gebräuchlich, s. W. Spiegelberg, Rec'Trav 28, 1906, 189-190; E. Lüddeckens, in: Demotische und koptische Texte, Papyrologica Coloniensia, II, Köln/Opladen 1968, 19-20 (27); S. Vleeming, in: LÄ III, 1209; M. Depauw, A Companion to Demotic Studies, Papyrologica Bruxellensia 28, 1997, 165-166.

Da hier und in Z. 9 für die Ausmaße des Baus jeweils die Folge $q 3 w-w s h-m d w$ gebraucht wird, kann mit $q 3$ w nur dessen Länge gemeint sein, nicht die Höhe, vgl. Wb V, 4,9; Wils on, A Ptolemaic Lexikon, 1045.

(18) Die obere Zahlenreihe hinter n'Tr ist weitgehend zerstört, aber der noch sichtbare Strich steht etwas weiter innen als der darunter, was eher für " als für "I' (so Daressy) spricht. Außerdem würde eine Breite von $51 / 2$ Ellen gut zu den vorhergehenden 11 Ellen passen (die Hälfte davon).

(19) S. u., Anm. 33.

(20) Zur Bezeichnung des Abschlusses von Bauarbeiten wird grh auch sonst verwendet, s. Wb V, 182,6. Zu 3 t „Hartstein“ vgl. man S. Aufrère, L'univers minéral dans la pensée égyptienne, I, BdE 105, 1991, 101-103; 110, n. 108. So verstanden wäre das grh $n$ w 3 h ${ }^{c} 3 t$ so etwas wie das Ende des „Rohbaus“ (des Verlegens der Steine, ohne Dekoration etc.).

Denkbar wäre aber auch, ${ }^{3} 3 t n f r y(t)$ als einen zusammengehörigen Ausdruck zu verstehen, eine Art „Schlussstein“. Dann müsste es heißen: „der Abschluss, nämlich das Legen des Schlusssteins: 2. Monat ... " Aber ein derartiger „Schlussstein" (es handelt sich ja nicht um ein Gewölbe) oder eine mit dem Verlegen des letzten Steins verbundene Zeremonie bei der Bauübergabe ist $\mathrm{m}$. W. aus anderen Zusammenhängen nicht bekannt.

(21) Daressy liest 3. Monat und Tag 6. Unter der Mondhieroglyphe oben in Kol. 7 glaubt man auf den ersten Blick in der Tat links noch einen weiteren Strich zu erkennen. Es dürfte sich aber eher um eine Beschädigung handeln, denn sonst wären die Abstände unregelmäßig und die ganze Zahl exzentrisch, etwas zu weit nach links gestellt. Bei der Tageszahl ist die (auch vom $\mathrm{Zu}-$ sammenhang geforderte) Lesung 9 jedenfalls wahrscheinlicher als Daressys 6. Die Zahlengruppe ist allerdings etwas unregelmäßig graviert (unterschiedlich lange Striche).

(22) Wörtlich „vollmacht“. Durch eine falsche Lesung ( $\curvearrowright$ statt $\urcorner$ ) hat Daressy sich und seinen Nachfolgern das Verständnis der gesamten Passage verbaut, vgl. etwa Gorre, Les relations du clergé égyptien et des Lagides d'après les sources privées, 55 , der sich zurecht über einen Monat mit 39 Tagen wundert.

(23) Daressy gibt statt dessen Jahr 4, denn wiederum ist die Jahreszahl beschädigt, man könnte 3, 4 oder auch 5 lesen. Tatsächlich sprechen die Spuren und v. a. ihre Stellung aber am ehesten für Jahr 3. Bei 4 wäre die Position exzentrisch, bei 5 die beiden äußeren Striche sehr schwach eingetieft. Bei der Tageszahl ist kaum noch eine Vertiefung zu erkennen. Daressys 1 ist gut möglich, aber 2 oder 10 auch nicht ausgeschlossen.

(24) Der Mann mit der Hand am Mund (signlist A2) ist relativ sicher, das $w h m$-Zeichen dagegen bestenfalls zu erahnen. Daressys Lesung $p \underline{d} . j$ ist auf jeden Fall ganz ausgeschlossen.

${ }^{(25)}$ Die (sicher vom Demotischen beeinflusste) Schreibung des Objektpronomens wj durch $\triangle \odot$ oder $\odot$ ist in dieser Zeit nicht selten, s. Jansen-Winkeln, Biographische und Religiöse Inschriften 154 (41); id., SAK 36, 2007, 65 (12). Man kann sie natürlich auch als separate (demotische) Form des Objektpronomens klassifizieren, vgl. D. Kurth, Einführung ins Ptolemäische, II, Hützel 2008, \ 49.

(26) Die Bezeichnung königlicher Bauten, und zwar - wie hier (Z. 2 und 8) - (zufällig?) ausschließlich in Luxor und Karnak, durch bj3jt „Wunderdinge“ o. ä. ist im Neuen Reich sehr häufig, s. E. Graefe, Untersuchungen zur Wortfamilie bj3-, Diss. Köln 1971, 101-104.

(27) Vgl. Anm. 16.

(28) Die Zahl neben dem ntr-Zeichen ist ziem-

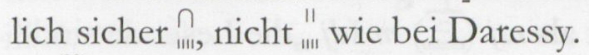

(29) Unter dem $r$ scheinen Reste von Strichen zu sein, aber ihre Anzahl ist sehr unsicher, am ehesten wohl 4 (Daressy gibt 6). 
Bei hn(w) wird es sich nicht um die Präposition „in“ handeln, denn nach der Maßangabe kann kaum eine Lageangabe folgen, die sich ja auf das gesamte Bauwerk beziehen müßte. Man sollte erwarten, dass $\underline{h} n w$ parallel zu dem folgenden hnw zu verstehen ist, das seinem Determinativ zufolge ein Nomen sein sollte.

${ }^{(30)}$ Die Lesung des entscheidenden ersten Zeichens (vor dem Doppelschilfblatt) ist sehr unsicher, vielleicht am ehesten प, und dann wäre wohl jwnyt zu lesen (vgl. dazu P. Spencer, The Egyptian Temple, London 1984, 63-67).

31) Falls die beiden etwas unförmigen Vertiefungen Schriftzeichen sind und keine Beschädigungen, wäre mit Daressy 6 zu lesen. Es ist allerdings auch möglich, dass die Stelle beschädigt ist. Den Spuren nach ist jedenfalls auch eine 10 über der 4, also eine Lesung 14, möglich.

(32) Die Lesung $h n w$ ist ziemlich sicher, nicht etwa wsht $(\stackrel{\square}{a})$ wie bei Daressy. Das wshZeichen in der gleichen Kolumne hat eine deutlich andere Form.

Bei dem folgenden Wort ist die Lesung jwnyt wieder alles andere als sicher, man könnte $z$. B. durchaus auch $\mathrm{DA}_{\mathrm{g}}$ lesen ( $\mathrm{zu}$ C $3 y t$ vgl. Wils on, A Ptolemaic Lexikon, 134-135). Der Zustand der Inschrift lässt nicht mehr als Vermutungen zu.

Zudem ist das Verständnis der ganzen Passage ganz unsicher: gegenüber dem ersten Baubericht in Z. 5-7 ist hier hinter den Maßangaben für Länge und Breite noch eine zusätzliche Angabe gemacht, deren Funktion fraglich ist. Vielleicht wird die Größe des neuerbauten Raumes mit irgendeinem bekannten Gebäude des Tempels verglichen, in dessen Nähe das „Goldhaus“ dann vermutlich erbaut war.

(33) Hier und in Z. 6 kommt offenbar der gleiche Ausdruck vor: $m \underline{d} w . f \underline{h} r \mathrm{XY} m h-n t r$ 9. In Z. 6 sieht das fragliche Element fast wie $\square$ aus, in Z. 9 fast wie $\overrightarrow{\square \square}$. Es könnte sich aber durchaus auch um die tierische Vorderpfote handeln (die in der Spätzeit sehr unterschiedliche Formen hat), und dann bietet es sich an, an beiden Stellen zu lesen. Das gemeinsame Element wäre dann $k 3 p(w)$,Dach“.

${ }^{(34)}$ Die Lesung der Zahl als 9 ist sicher, aber darunter sind noch Spuren, und auch der Raumverteilung nach sollte man eigentlich noch eine halbe Gruppe erwarten. Ergänzt man hier noch 111, würde die „Tiefe“ des Raumes (9 1/3) 2/3 seiner Länge (und Breite ?) entsprechen (14:9 1/3). Allerdings: die Spuren scheinen nicht wirklich dazu zu passen.

(35) Die Form des ${ }^{3} t$ (?) ist deutlich anders als in Kol. 6, vermutlich aber nur etwas entstellt.

(36) Die Ergänzung zu 16, den Spuren und dem Platz nach möglich, ergibt sich aus der Gesamtdauer von 7 Monaten und 10 Tagen $(=220$ Tage).

(37) Oder ,der das ganze Land erblickt“.

(38) Lesung nicht sicher, statt $r d i w n$ wäre auch möglich. Die folgenden 1 1/2 Gruppen sind mir unverständlich.

(39) Zum häufigen Zusammenhang von $w n-h r$ und $w d \underline{d} t$-Auge s. A. Lohwasser, Die Formel ,Öffnen des Gesichts', Wien 1991, 37-39.

${ }^{(40)}$ Entsprechende Aussagen sind in der Spätzeit nicht selten, z. B. auf den Statuen Kairo JE 37196 (Jansen-Winkeln, CdE 78, 2003, 3738, Z. 5-6: jnk hm ... z3 hm pr.k „,ich bin ein Diener ... der Sohn eines Dieners deines Hauses"), London BM $1132+1225$ (unpubl.): jnk hm z’ hm m pr.t (dto.), Kairo CG 672, linke Seite, Z. 4 (R. el-Sayed, Documents relatifs à Sais et ses divinités, BdE 69, 1975, 86: jnk hm z’ hm $n$ pr $p n$,ich bin ein Diener, der Sohn eines Dieners dieses Hauses"); Würfelhocker Linköping 102, Vorderseite, Z. 4-5 (G. Björkman, The Smith Collection of Egyptian Antiquities, Uppsala 1971, 17) jnk hm.kz3 n pr.k dr jtjw.f, ich bin dein Diener, ein Sohn deines Hauses von seinen Vätern her").

(41) Man sollte eher die 3. Person erwarten (,ein Diener von dir, indem er gehört" = ,der gehört").

(42) Lesung $s{ }^{\mathcal{C}} 3$ ist $s e b r$ unsicher.

(43) Die Lesung $\mathbb{F r k}^{\text {ro }}$ ist relativ sicher, die Gruppe davor keineswegs. Falls das erste Zeichen ein $f$ sein sollte, könnte man an ${ }^{C} n h-t 3-h s 3 t$ (oder auch ${ }^{c} n h-t 3$-jht/jht $)$ denken, vgl. etwa PN I, $66,4\left({ }^{(} n h-h s^{3} 3 t\right)$. Aber es gibt auch noch andere Möglichkeiten. Der Name der Mutter bleibt daher vorläufig fraglich.

(44) Nur das Zeichen $n s / j m j-r$ scheint einigermaßen sicher. Vermutlich stand im folgenden der erste Titel ihres Vaters Jmn-htp. Es ist allerdings nicht völlig auszuschließen (wenn auch 
unwahrscheinlich), dass am Ende von Kol. 14 der Name ihres Vaters stand sowie $z$ 3 „Sohn des"; dann wäre Jmn-htp nicht ihr Vater, sondern der Großvater.

(45) Mit dem Namen des Ns-p3wtj-t3wj ist die Filiation der Mutter des Sohnes (also der Ehefrau des Sprechers) abgeschlossen, denn es folgt ja die Präposition $m j$. Dass diese Filiation überhaupt angeführt wird (in dieser Zeit bei der Mutter eher ungewöhnlich) hat sicher den Grund, dass ihr Großvater den besonders ehrenvollen Posten eines Hohenpriesters des Amun bekleidete. Zu diesem hm-ntr tpj (n Jmn) Ns-p3wtj-t3wj und seiner zeitlichen Stellung s. den Anhang.

(46) Die Phrase ist offenbar parallel zu der von Kol. 14 (oben): Der Sprecher erbittet zunächst göttliche Gnaden für sich „entsprechend dem, was ich getan habe" ( $f q 3 w n . j m c^{c} c^{c} w q^{3 j}$... $m$ j jrt.n.j) und sagt dann, dass sein Sohn angesehen ist, weil er entsprechend seinem Vater gehandelt

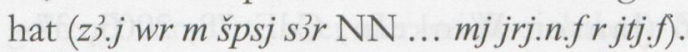

(47) Sehr unsicher, es könnte irgendein mythologischer Vergleich zwischen den Taten des Sohnes und des Harsiese beabsichtigt sein; die folgenden Gruppen sind mir unverständlich.

(48) Zur Erklärung der 96 Jahre s. u. (Inhalt und Zweck der Inschrift). Die Zahl nach hrdw ist wohl am ehesten zu einer runden Zahl wie 20 oder auch 12 zu ergänzen, also [ $\mathrm{n}] \cap$ oder ["]ก. Bei der Anzahl der Kinder scheint es keine Idealvorstellung (wie beim Lebensalter) zu geben.

(49) Der gleiche Anruf in Z. 3-4.

(50) 므을 ist eine (in diesem Zusammenhang ungewöhnliche) orthographische Variante von $\sqrt{\pi K}$, vgl. Kol. 4.

(51) $t p$ vor $n f r$ nicht sicher, aber möglich. Der Rest der Kolumne (ca. fünf Gruppen) ist kaum lesbar, ich kann ihm jedenfalls keinen Sinn abgewinnen. Man sollte so etwas erwarten wie „möget ihr veranlassen, dass ist (oder bleibt) (mein Sohn in der Gunst des Großen Widders ..." о. ä.).

(52) Die Lesung $j r w^{c} b n b$ ist schon nicht sicher, und vom Rest der Kolumne sind nur einzelne Zeichen erkennbar. Das $f$ unten über dem (mutmaßlichen) $z 3$ könnte dem Zusammenhang

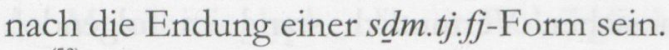

(53) Falls die Gruppe unter $f q$ 3 (mit Daressy) $\underset{\sim}{\sim} \mathrm{zu}$ lesen ist, müsste man vor $n b$ ntrw ein $n$ ergänzen. Diese Lesung ist aber nicht sicher, man könnte die beiden waagerechten Striche auch als $\underset{-\infty}{\longleftarrow}$ interpretieren. Dann müsste man „der Herr der Götter möge ihn belohnen“ ( $f q$ ? $s w)$ übersetzen.

(54) Auch hier ist wieder der untere Teil der Kolumne (unter špsj sir) bis auf die letzte Gruppe kaum lesbar. Man würde so etwas wie „im Westen von Theben" erwarten.

(55) Die Gruppe unter jr(r) sieht fast wie (o. ä.) aus, anders gesagt, das obere Zeichen setzt sich nach links unten fort. Diese Fortsetzung wird aber zufällig sein, denn sie reicht etwas weiter nach links als die anderen Zeichen dieser Kolumne. Es wird daher (mit Daressy) zu lesen sein. Andernfalls müsste man es als „entsprechend dem, was der, den er aufgezogen hat, sein ältester Sohn, getan hat" verstehen.

\section{Inhalt und Zweck der Inschrift}

Aus der vorangegangenen Behandlung dieser Inschrift ist wohl deutlich geworden, wie vieles darin noch unklar ist, da zahlreiche Zeichen kaum zu identifizieren sind. Dennoch ist der Inhalt in den Grundzügen klar:

In dem Abschnitt, wo von den Tätigkeiten des Verfassers als „Baumeister" die Rede ist (Kol. 5-10), werden drei Vorgänge berichtet:

a) Im Jahr 1 (1. Achet, Tag 1) unter Alexander dem Großen wird der Bau eines Goldhauses (pr nbw) für den Gott Amenope begonnen, also sicher im Tempel von Luxor, und in 39 Tagen (2. Achet, Tag 9) abgeschlossen. Auch wenn das Datum des Beginns der Arbeiten (Neujahrstag im ersten Jahr Alexanders) vermutlich fiktiv ist, gibt es kaum Grund, an der Angabe der Bauzeit selbst zu zweifeln.

b) Im Jahr 3 (3. Achet, Tag 1) unter Philipp Arrhidäus (11 Jahre später, 321) wird $c_{n h-p} 3$ - hrrd (von Amun!) zum Baumeister, ,in seinem Haus“ (vermutlich Karnak) ernannt.

c) Gut zwei Monate später, am 1. Peret, Tag 7 im selben Jahr, beginnt er den Bau eines Goldhauses für Amonrasonther, also in Karnak, und dieser Bau wird in 220 Tagen (7 Monaten und ein Drittel) am 4. Schemu, Tag 16 beendet. 
Die beiden Bautexte (a und c) haben folgende Struktur:
a) Datum:
rnpt-zp tpj 3ht, $1 \mathrm{hr} h \mathrm{hm}(\mathrm{n})$ nswt-bjt 3lksndrs

Baubeginn:

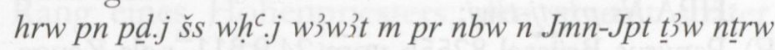

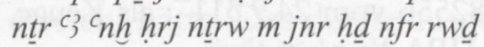

Maße:

$q 3 w . f$

wsh.f

mh-ntr 11

$m \underline{d} w$. fhr kjpw mh-ntr 9

Bauende:

grh $n$ wih c3t nfry(tr) 2. 3ht, $9 \mathrm{~m}$ rnpt tn

Bauzeit:

mh hrw 39

c) Datum:

tpj prt, $7 \mathrm{~m}$ rnpt tn (rnpt-zp 3 Prpys)

Baubeginn:

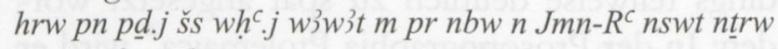
m jnr nfr rwd

Maße:

q3w.f mh-ntr $14 \quad r^{3} 4$ hnw jwnyt

wsh.f mh-ntrit (?) hnw jwnyt(?)

$m d w$. f hr kjpw mh-ntro $9(1 / 3$ ? $)$

Bauende:

grh $\underline{h} r^{c} 3 t n f r y(t r) 4 . s ̌ m w, 1[6] m$ rnpt tn

Bauzeit:

r $3 b d 71 / 3$

Es handelt sich in beiden Fällen um rechteckige Räume von recht bescheidener Größe: das Goldhaus im Luxortempel hat die Maße $11 \times 5,5($ ? $) \times 9$,Gottesellen“, das Goldhaus in Karnak ist etwas größer, vermutlich $14 \times 14(?) \times 9(, 33$ ? $) \quad$ „Gottesellen “" ${ }^{12}$. Es sind kleinere Arbeiten, wie es in dieser Zeit und bei dieser Person (die keineswegs zu den höchsten Rängen der Hierarchie gehört) nicht anders zu erwarten ist. Beide Bauten sind aus Sandstein errichtet, daher sicher im Tempel selbst gelegen, nicht in den Magazinbereichen. Die Beschreibung scheint aber auf keine der nicht sehr zahlreichen noch in die Argeadenzeit datierbaren Bauten in Luxor und Karnak zu passen. Da aber unter Alexander bekanntlich ein Sanktuar im

\footnotetext{
${ }^{12}$ Ebenso lang $(52,5 \mathrm{~cm})$ wie die ältere „einfache“ Elle $(m h)$, s. LÄ III, 1209.
}

Luxortempel gebaut wurde ${ }^{13}$ und unter Philipp Arrhidäus in Karnak ${ }^{14}$, wäre es gar nicht erstaunlich, wenn in diesem Rahmen auch ein neues „Goldhaus" errichtet wurde. Der Priester ${ } n h-p$ 3hrd dürfte daher jeweils mit einer Nebenaufgabe innerhalb eines größeren Bauprojekts betraut worden sein.

In einem größeren Rahmen gehören diese Bauten sicherlich zur Wiederaufnahme der Bautätigkeit an den Tempeln nach der zweiten Perserherrschaft, als eine Art Fortsetzung des Bauprogramms der 30. Dynastie. Daher wohl auch die (vermutlich fiktive) Datierung des Baubeginns in Luxor auf den ersten Regierungstag Alexanders.

In der zweiten Hälfte der Inschrift ist nicht mehr von Bauten die Rede. Dort zeigt sich, dass der Verfasser mit dieser kleinen Inschrift nicht allein den Zweck verfolgt, seine eigenen Verdienste herauszustellen. Sie sind vielmehr (auch) eine Art Einleitung und Voraussetzung zu einer Fürbitte für seinen Sohn, sicherlich mit dem Ziel, ihn später als Nachfolger einsetzen zu lassen. Dieser Sohn soll (weitere) 96 Jahre leben (Kol. 16). Da später für diejenigen, die ihm behilflich sein sollten, die übliche Idealzeit von 110 Lebensjahren $^{15}$ erbeten wird (Kol. 19), war dieser Sohn zum Zeitpunkt des Verfassens der Inschrift vermutlich 14 Jahre alt, d. h. im kritischen Alter für eine beginnende Priesterkarrie$\mathrm{re}^{16}$, nach Abschluss der „Schulausbildung“. Vermutlich wird deshalb auch die Eigenschaft des $s 3 r$ (gebildet o. ä., s. Anm. 8 zur Übersetzung) so sehr betont: denn die Kenntnis alter Schriften und des Hieratischen war ja in dieser Zeit eine wichtige Voraussetzung für eine höhere Priesterlaufbahn.

${ }^{13}$ PM II 2 , 324-326; M. Abd el-Raziq, Die Darstellungen und Texte des Sanktuars Alexanders des Großen im Tempel von Luxor, AV 16, 1984.

${ }^{14} \mathrm{PM} \mathrm{I}^{2}, 99-102$.

${ }^{15}$ S. Janssen, OMRO 31, 1950, 33-44.

${ }^{16}$ Vgl. W. Otto, Priester und Tempel im hellenistischen Agypten, I, Leipzig/Berlin 1905, 211; 214-215; Staatliche Museen zu Berlin, Griechische Urkunden, Bd. 5, Berlin 1934, § 91. 


\section{Die Familie der Frau des $c_{n h-p} 3-\underline{h r d}$}

In der Inschrift werden außer dem Verfasser $c_{n h-p}$ - $h r d$ auch sein Vater Jrt-Hr-r.w, seine Mutter T3-hjj-bj3t und sein Sohn Jrt-Hr-r.w erwähnt. Auf der von $c_{n h-p}$ - $h r d$ gestifteten Statue seines Vaters Kairo JE 37989 werden zudem noch die Eltern dieses Vaters namens $N s-j n j-h r t$ und $N b$ 3ht genannt ${ }^{17}$.

In Z. 14-16 der Luxor-Inschrift wird anlässlich der Vorstellung des Sohnes und der Fürbitte für ihn zusätzlich die. Abstammung seiner Mutter, also der Ehefrau des ${ }^{c} n h-p 3-\underline{h} r d$ genannt und über zumindest zwei Generationen weitergeführt. Der älteste erwähnte Vorfahr ist der Großvater (weniger wahrscheinlich der Urgroßvater, s. Anm. 44 zur Übersetzung) der Mutter, ein Hoherpriester des Amun namens Ns-p3wtjt3wj (Spotous).

Die Hohenpriester der 30. Dynastie bzw. der frühen Ptolemäerzeit sind in den letzten Jahrzehnten wiederholt behandelt worden, u. a. von J. Quaegebeur ${ }^{18}$, H. De Meulenaere ${ }^{19}$ und M. Depauw ${ }^{20}$. Die relevanten Zeugnisse dafür sind folgende ${ }^{21}$ :

A) Kanopenkasten Brüssel E.7624 : gehört dem HPA Ns-piwtj-tiwj, Sohn des Wsjr-wr und der $N h m-s j-R^{c} t-t i w j$

B) Situla Louvre N.908 $8^{23}$ : gehört dem HPA $W s j r-w r$, Sohn des Ns-p3wtj-t3wj (mj nn) und der Nhm-sj-Rt $R^{c}$ t3wj;

${ }^{17}$ Jansen-Winkeln, Biographische und religiöse Inschriften, 179-180.

${ }^{18}$ In: Vleeming (ed.), Hundred-Gated Thebes, $155-158$.

${ }^{19}$ In: W. Clarysse et al. (edd.), Studies Dedicated to the Memory of Jan Quaegebeur, II, 1118-1123.

${ }^{20}$ The Archive of Teos and Thabis from Early Ptolemaic Thebes, MRE 8, 2000, 178-180.

${ }^{21}$ Bezeichnungen A-H nach Depauw, op. cit., 180; HPA = Hoherpriester des Amun (hm-ntr tpj $n$ Jmn).

${ }^{22}$ Quaegebeur, in: Vleeming (ed.), HundredGated Thebes, 157.

${ }^{23}$ P. Pierret, Recueil d'inscriptions inédites du Musée égyptien du Louvre, II, Paris 1878, 113-118; M. Étienne, Les portes du ciel, Ausstellungskatalog Louvre, Paris 2009, 251 (Nr. 201); W. Peremans/ E. Van 't Dack, Prosopographia Ptolemaica, IX, Löwen 1981, 92-93 (Nr. 5669).
C) Stele Louvre N.2099 $9^{24}$ : gehört dem HPA $W_{s j r}$-wr, Sohn des HPA Ns-piwtj-tiwj und der $N h m-s j-R^{c} t-$ t3wj;

D) Papyri London BM 10530 und 10535 aus dem Jahr 283 (10530) bzw. $279(10535)^{25}$ : genannt wird jeweils ein Schreiber Wsjr-wr, Sohn eines HPA Ns-p3wtj-t3wj;

E) Papyrus Brüssel $8255 \mathrm{~b}$ vom 24.9.311 ${ }^{26}$ : als Zeuge unterschreibt ein HPA $N s-p 3 w t j-t 3 w j$;

F) Würfelhocker Kairo JE $37146^{27}$ : gehört dem HPA Ns-p3wtj-t3wj, Sohn eines hm-ntr Wsjr-wr und einer $M w t-j r j-d j-s$ (s. u., Anhang);

G) Bau- und Bittinschrift in Luxor (hier behandelt): der Großvater der Ehefrau des Verfassers ${ }^{2} n h-p$; $h r d$ ist ein HPA Ns-piwtj-t3wj;

H) Stele Louvre E. $15565^{28}$ : gehört einer Ns-Hnzw, Tochter eines HPA $W_{s j r}$-wr und der $T 3-h 33 b 3 s$.

In den oben erwähnten Arbeiten ist auch der in der hier behandelten Inschrift bezeugte $N s-p 3 w t j-t 3 w j$ berücksichtigt worden. Er ist allerdings teilweise deutlich zu spät angesetzt worden: In der Prosopographia Ptolemaica ${ }^{29}$ wird er mit dem gleichnamigen Hohenpriester identifiziert, der in dem Papyrus Brüssel 8255b am 24.9.311 als Zeuge unterschreibt ${ }^{30}$, ebenso in den von Quaegebeur und De Meulenaere vorgeschlagenen Stammbäumen. Der Hohepriester $N s-p 3 w t j-t 3 w j$ aus der Luxor-Inschrift aus dem Jahr 320 ist aber (mindestens) zwei Generationen älter als ihr Verfasser ${ }^{c} n h-p$ j- $h r d$ bzw. dessen Frau, die ihrerseits schon einen schriftkundigen, also nicht mehr ganz kleinen Sohn haben ${ }^{31}$. Daher ist es sehr unwahrscheinlich (wenn auch nicht völlig ausgeschlossen), dass ihr Großvater noch 9 Jahre später als Zeuge und amtierender Hoherpriester erscheint. In der Genealogie

${ }^{24}$ Pierret, Recueil d'inscriptions, II, 121-123; Abd el Hamid Zayed, ASAE 56, 1959, pl. II.

${ }^{25}$ Depauw, The Archive of Teos and Thabis from Early Ptolemaic Thebes, 178-179.

${ }^{26}$ Depauw, op. cit., 168-180.

${ }^{27}$ De Meulenaere, in: W. Clarysse et al. (edd.), Studies Dedicated to the Memory of Jan Quaegebeur, II, 1120-1121.

${ }^{28}$ P. Munro, Die spätägyptischen Totenstelen, ÄF 25, 1973, 230; Taf. 13, Abb. 47.

${ }^{29}$ Peremans/Van 't Dack, Prosopographia Ptolemaica, IX, 114-115, Nr. 5812.

${ }^{30}$ S. Depauw, The Archive of Teos and Thabis, $168-169$.

${ }^{31}$ War der Sohn 14 Jahre alt (s. o.), kann die Mutter zu diesem Zeitpunkt nicht viel jünger als 30 Jahre gewesen sein. 
Quaegebeurs käme hinzu, dass der Sohn dieses $N s-p 3 w t j-t 3 w j$ namens $W s j r-w r$ (eine Generation älter als der Verfasser der Luxorinschrift und dessen Frau!) dann in den Jahren 283 bzw. 279 bezeugt wäre ${ }^{32}$, aber noch (immer) nicht im Rang eines Hohenpriesters, den er erst später erreicht hätte. Das dürfte ausgeschlossen sein.

In der Genealogie De Meulenaeres ist der Wsjr-wr von 281/277 nicht der Sohn, sondern der Urenkel des Hohepriesters $N s-p 3 w t j-t 3 w j$ aus der Luxor-Inschrift. Der Urgroßvater wäre dann 311 als Zeuge aufgetreten, sein Urenkel nur 28 Jahre später. Das ist möglich, aber nicht sehr naheliegend. Überdies ist auch die in De Meulenaeres Stammbaum angesetzte dreimalige Abfolge $W s j r-w r-N s-p 3 w t j-t 3 w j$ (also über 6 Generationen), wovon die letzten 5 jeweils Hohepriester gewesen wären, recht unwahr- scheinlich. Dieser letztere Einwand trifft auch die genealogische Rekonstruktion von Depauw. Sie ist zwar ansprechender als die anderen Stammbäume, da sie den $N s-p 3 w t j-t 3 w j$ der Luxor-Inschrift zwei Generationen vor dem im Jahr 311 belegten gleichnamigen Hohenpriester ansetzt. Aber auch in diesem Stammbaum hätten wir über 6 Generationen einen Vater $W_{s j}$ r$w r$ mit einem Sohn Ns-p3wtj-t3wj, von denen dann Nr. 1 und 4-6 Hohepriester gewesen wären.

Mir scheint eine Rekonstruktion wie in Abb. 1 wahrscheinlicher ${ }^{33}$. In diesem Stammbaum ist der HPA Wsjr-wr von Beleg $\mathrm{H}$ (Vater der Ns-Hnzw) mit dem der Belege A-C identifiziert worden. Ihre hauptsächlichen Titel sind (nahezu) identisch (neben HPA noch

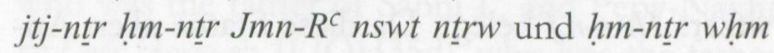

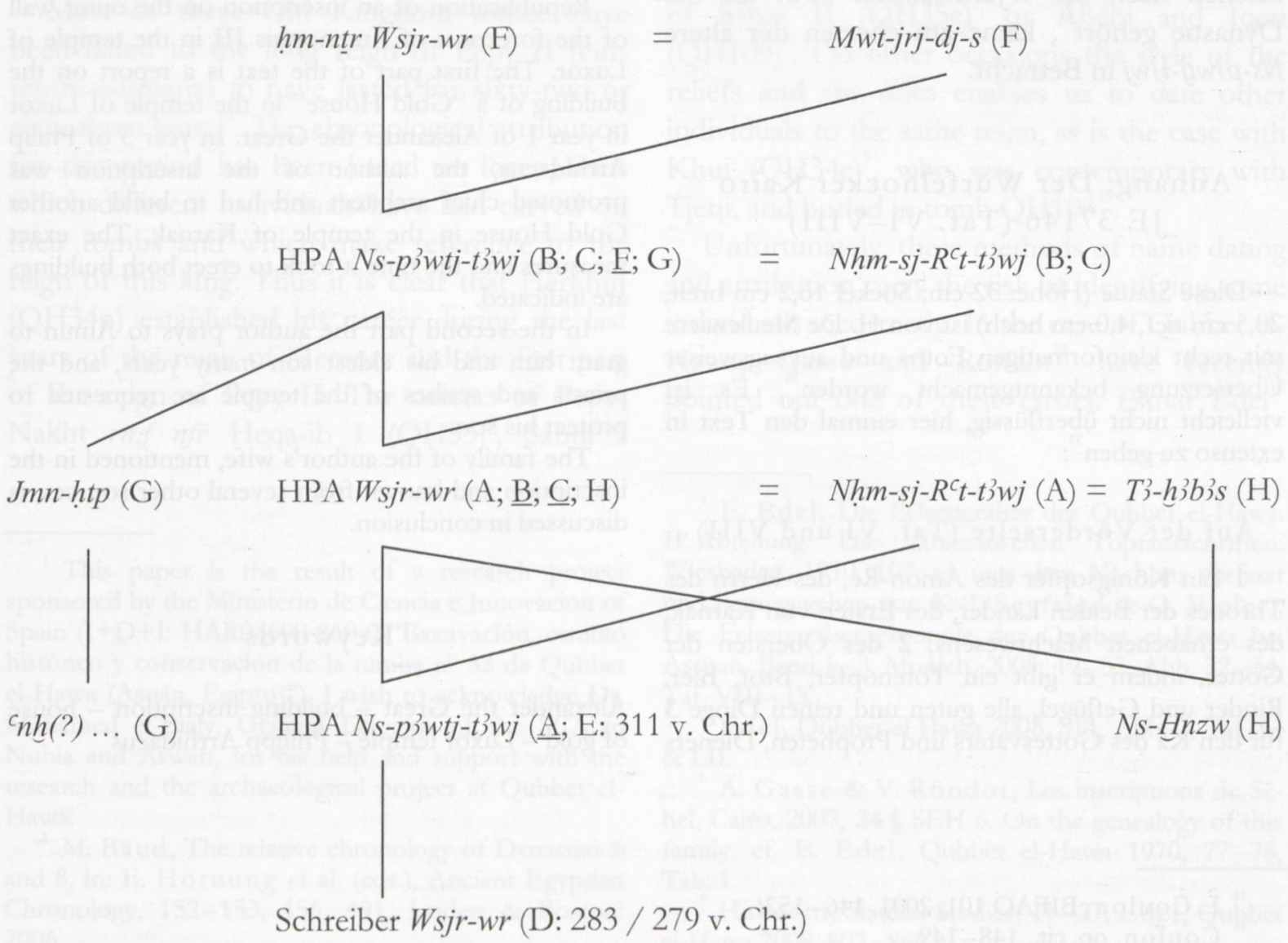

Abb. 1. Die Familie der Frau des $c_{n} \underline{h}-p j-\underline{h} r d$.

${ }^{32}$ In den Papyri BM 10530 und 10535, s. Depauw, op. cit., 178-179.
${ }^{33}$ Die Belege, deren Besitzer die jeweilige Person ist, sind unterstrichen. 
$n$ Jmn (C) bzw. ḥm-nțr whm n p3 nțr (H). Daneben führt $W_{s j r-w r}$ auf $\mathrm{B}$ und $\mathrm{C}$ noch eine Menge spezieller und z. T. seltener Priestertitel, und das gleiche gilt von $\mathrm{H}$ : die Titel sprechen eher für eine Identifizierung als dagegen. Man müsste allerdings annehmen, dass der HPA Wsjr-wr Kinder von zwei Frauen gehabt hätte, aber das ist kaum eine besonders fernliegende Möglichkeit.

Auf diese Weise hätten wir nur eine sich über drei Generationen erstreckende Abfolge der Hohenpriester Ns-p3:wtj-t3wj - Wsjr-wr - Nsp3wtj-t3wj, und der in der Luxor-Inschrift erwähnte HPA Ns-p3wtj-t3wj hätte zwei Generationen vor dem im Jahr 311 bezeugten gelebt.

Auf der Statue Kairo JE $37343^{34}$ ist ebenfalls ein HPA Ns-pjwtj-t3wj als Vater des Statuenbesitzers Hr.s-n.f belegt. Da diese Statue allen Anzeichen nach ins 4. Jahrhundert bzw. die 30 . Dynastie gehört ${ }^{35}$, käme am ehesten der ältere Ns-p3wtj-t3wj in Betracht.

\section{Anhang: Der Würfelhocker Kairo JE 37146 (Taf. VI-VIII)}

Diese Statue (Höhe: $32 \mathrm{~cm}$; Sockel 16,2 cm breit, $20,5 \mathrm{~cm}$ tief, $4,0 \mathrm{~cm}$ hoch) ist von $\mathrm{H}$. De Meulenaere mit recht kleinformatigen Fotos und auszugsweiser Übersetzung bekanntgemacht worden ${ }^{36}$. Es ist vielleicht nicht überflüssig, hier einmal den Text in extenso zu geben ${ }^{37}$ :

\section{Auf der Vorderseite (Taf. VI und VIII)}

1 Ein Königsopfer des Amon-Re, des Herrn des Thrones der Beiden Länder, des Ersten von Karnak, des erhabenen Machtwesens, 2 des Obersten der Götter, indem er gibt ein Totenopfer, Brot, Bier, Rinder und Geflügel, alle guten und reinen Dinge $\mathbf{3}$ für den Ka des Gottesvaters und Propheten, Dieners der ,Weißen', Dieners des Horus, des Vorstehers der Propheten in Theben, 4 des Öffners der Türflügel des Himmels, des Ersten Propheten des Amun 5 Ns$p 3 w t j-t 3 w j$, des Sohnes des Propheten $W$ sjr-wr; seine Mutter ist Mwt-jrj-dj-s.

\section{Auf dem Rückenpfeiler (Taf. VII und VIII)}

1 Der Stadtgott des Gottesvaters (und Propheten), Dieners der ,Weißen', Dieners des Horus, des Vorstehers der Propheten in Theben, des Ersten Propheten des Amun Ns-p3wtj-t3wj, des Sohnes des Propheten $\mathbf{2}$ Wsjr-wr ist hinter ihn gesetzt worden, gegenüber seinem $\mathrm{Ka}$, in seiner Gegenwart. $\mathrm{Er}$ ist ein mit einem (solchen) Pfeiler Versehener, ewig, ewig.

\section{Summary}

Republication of an inscription on the outer wall of the forecourt of Amenophis III in the temple of Luxor. The first part of the text is a report on the building of a "Gold House" in the temple of Luxor in year 1 of Alexander the Great. In year 3 of Philip Arrhidaeus, the author of the inscription was promoted chief architect and had to build another Gold House in the temple of Karnak. The exact measures and the time it took to erect both buildings are indicated.

In the second part the author prays to Amun to grant him and his eldest son many years, and the priests and scribes of the temple are requested to protect his son.

The family of the author's wife, mentioned in the inscription and known from several other sources, is discussed in conclusion.

\section{Keywords}

Alexander the Great - building inscription - house of gold - Luxor temple - Philipp Arrhidaeus
${ }^{34}$ L. Coulon, BIFAO 101, 2001, 146-152.

${ }^{35}$ Coulon, op. cit., $148-149$.

${ }^{36}$ In: W. Clarysse et al. (edd.), Studies Dedicated to the Memory of Jan Quaegebeur, 1120-1121; vgl. auch Legrain, ASAE 7, 1906, 41 (Nr. 465).

${ }^{37}$ Die Fotos sind im Jahr 2000 vom Museum Kairo aufgenommen worden. Für die Genehmigung zur Veröffentlichung danke ich dem damaligen Generaldirektor Dr. M. A. H. Shimy. 


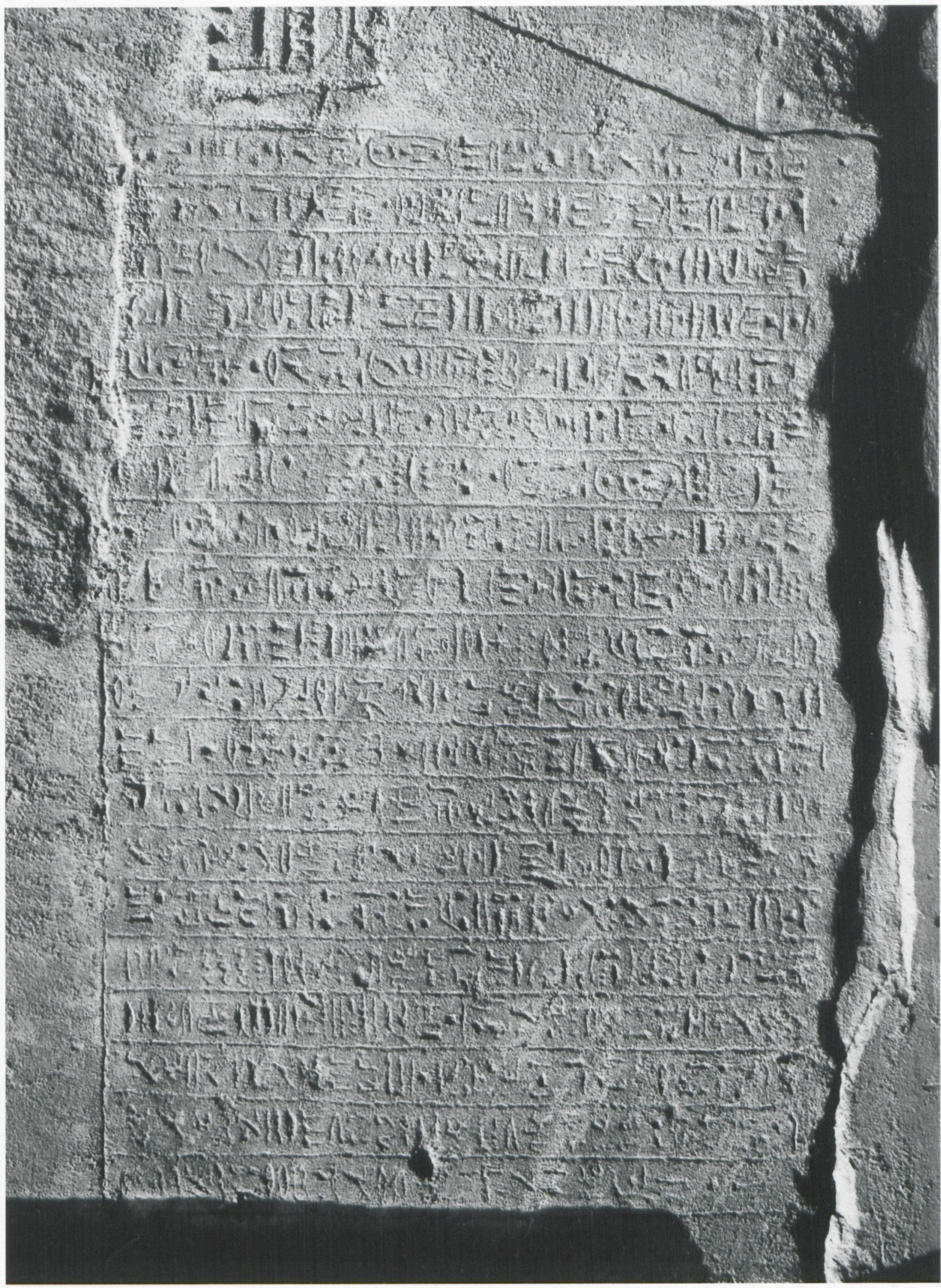

Inschrift an der Außenwand des Luxortempels (zu Jansen-Winkeln, Bau- und Bittinschrift). 
TO

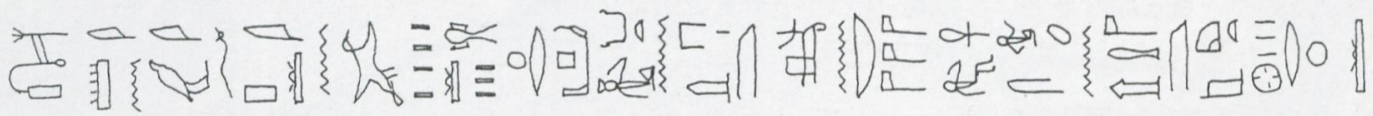

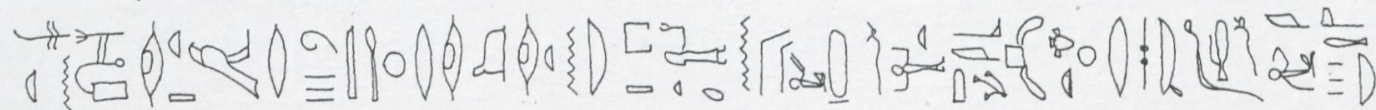

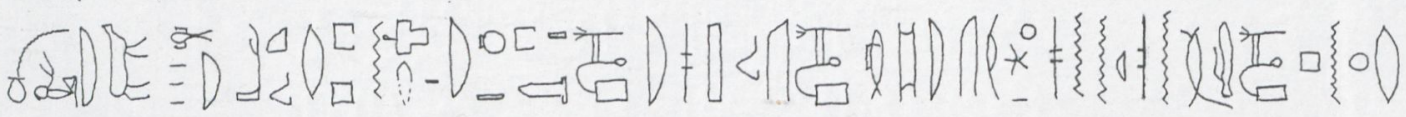

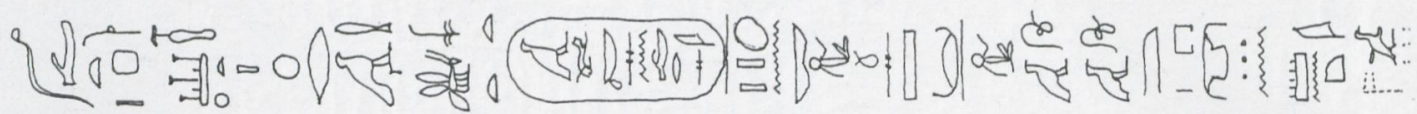

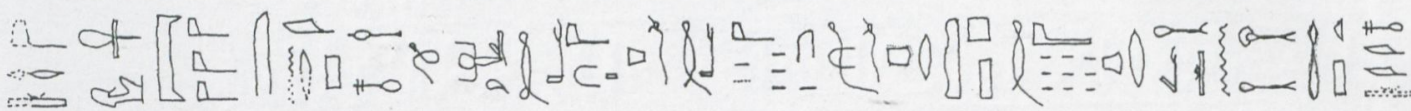

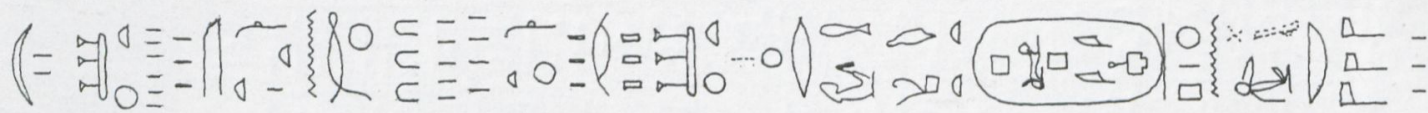

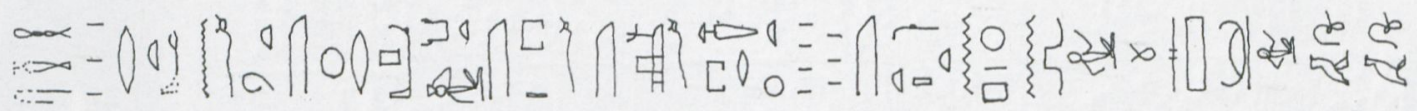
1 I. $00 y-a 0 \&$ a d a

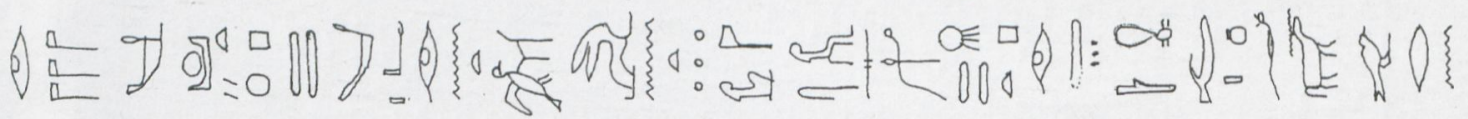
次/ Qर

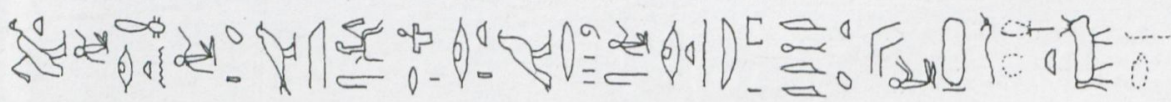

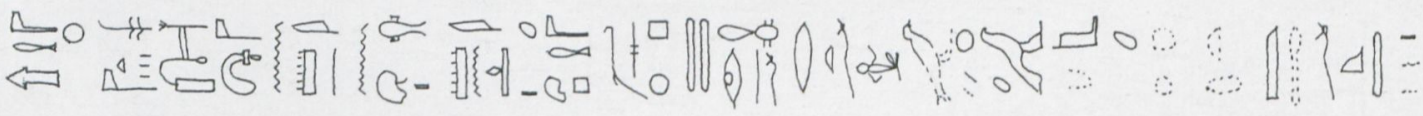

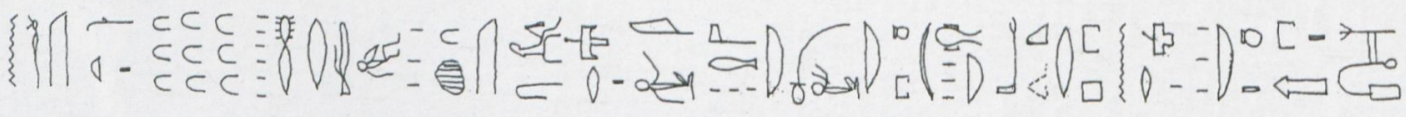

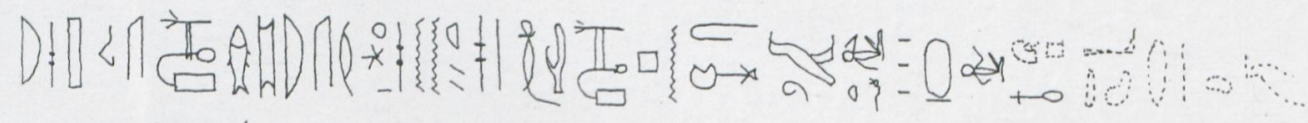

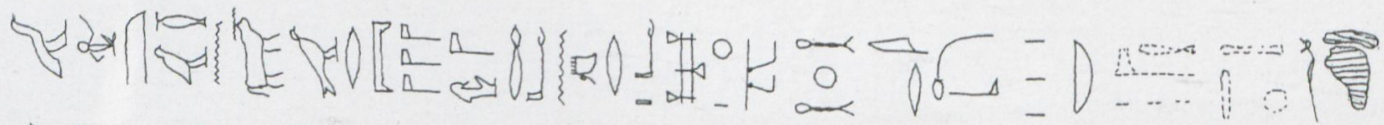

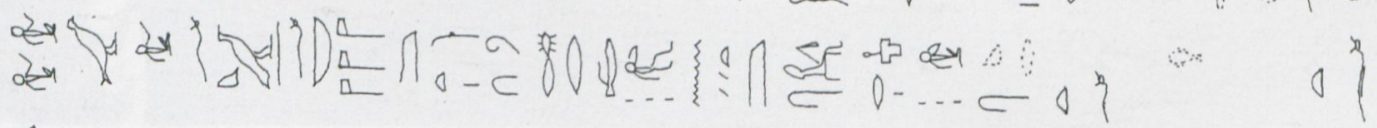

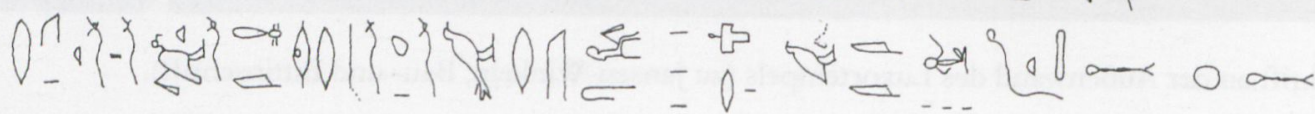




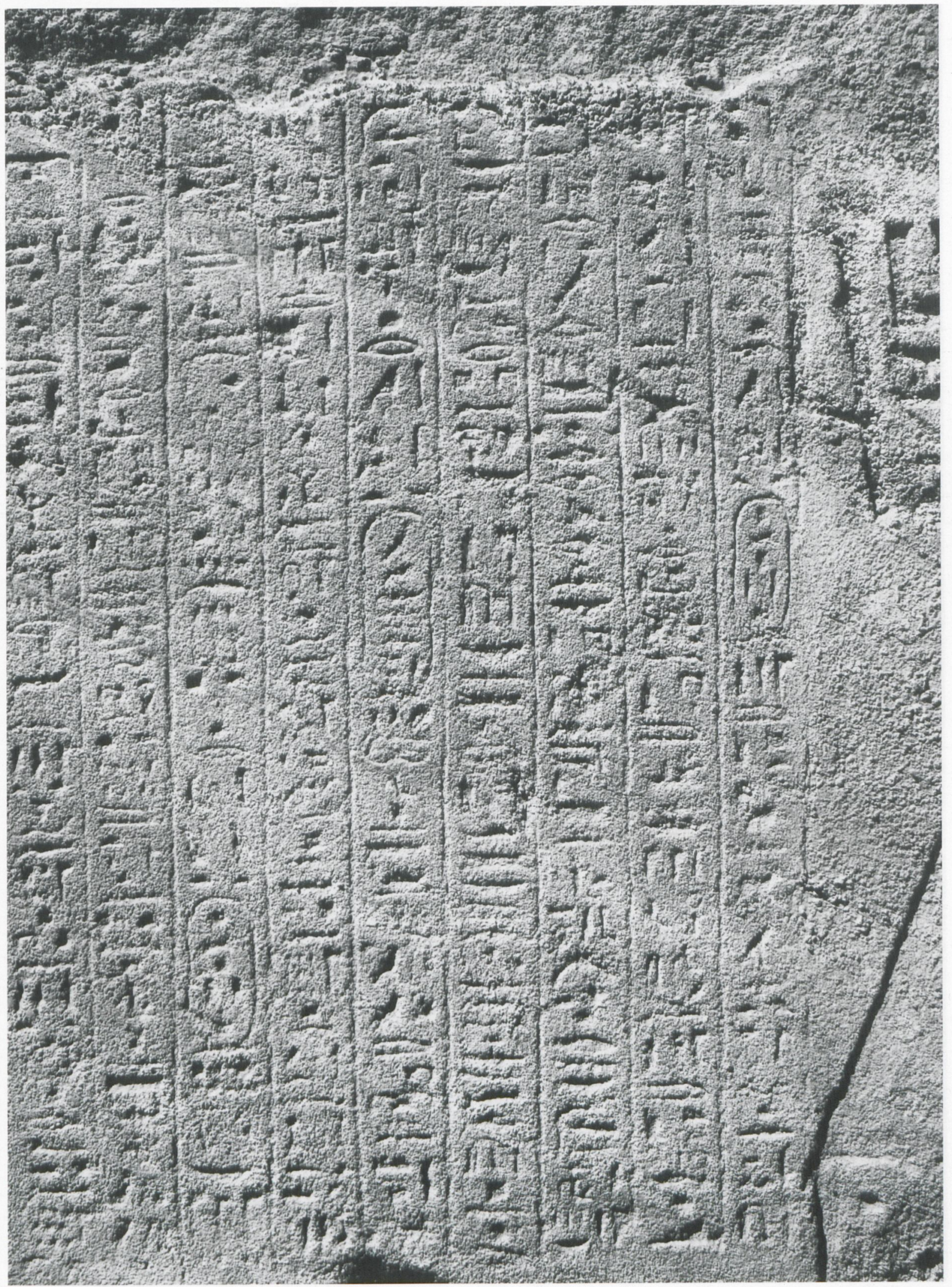

Inschrift an der Außenwand des Luxortempels, rechts (Z. 1-9) (zu Jansen-Winkeln, Bau- und Bittinschrift). 


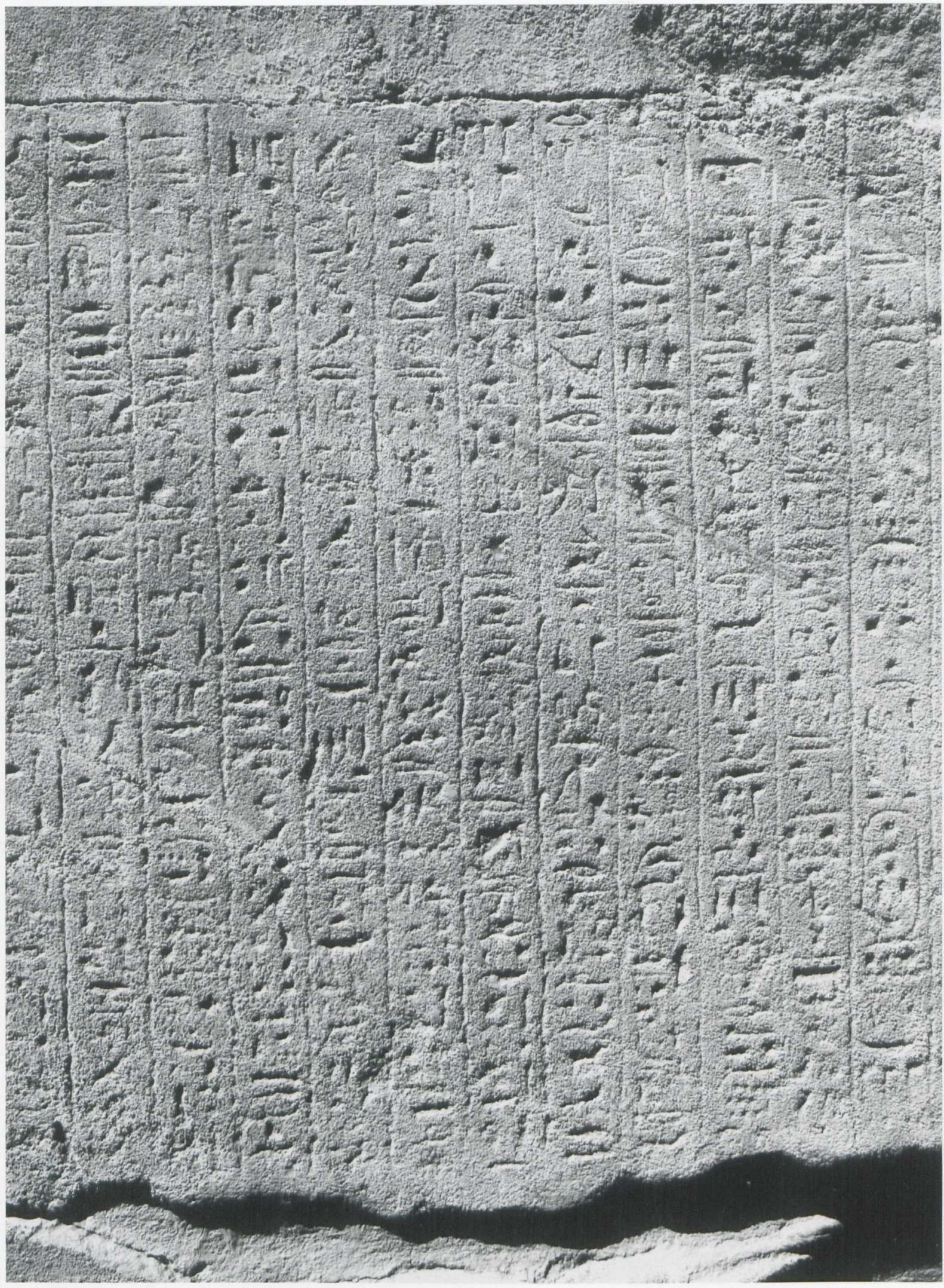

Inschrift an der Außenwand des Luxortempels, Mitte (Z. 7-17) (zu Jansen-Winkeln, Bau- und Bittinschrift). 


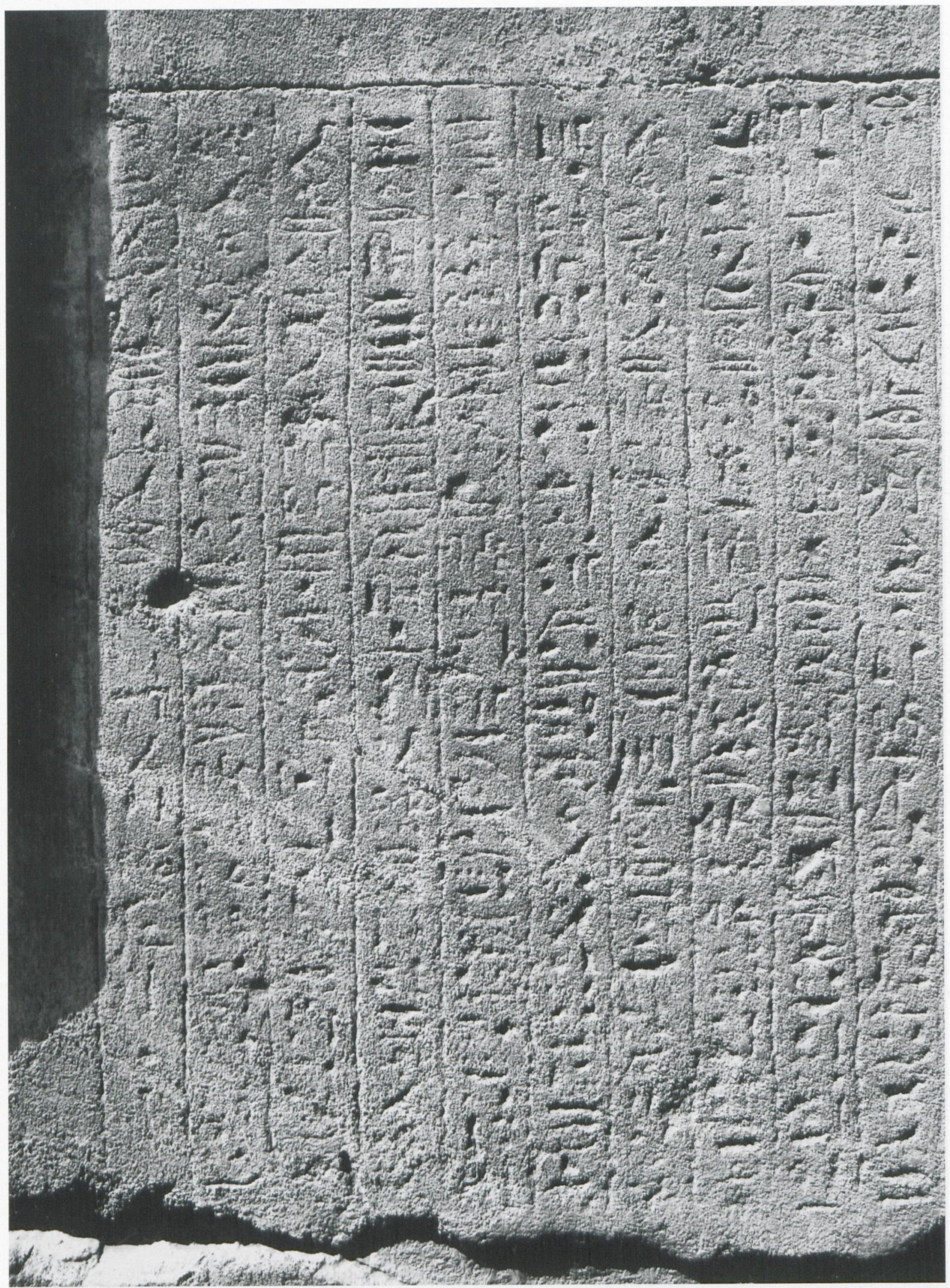

Inschrift an der Außenwand des Luxortempels, links (Z. 11-20) (zu Jansen-Winkeln, Bau- und Bittinschrift). 


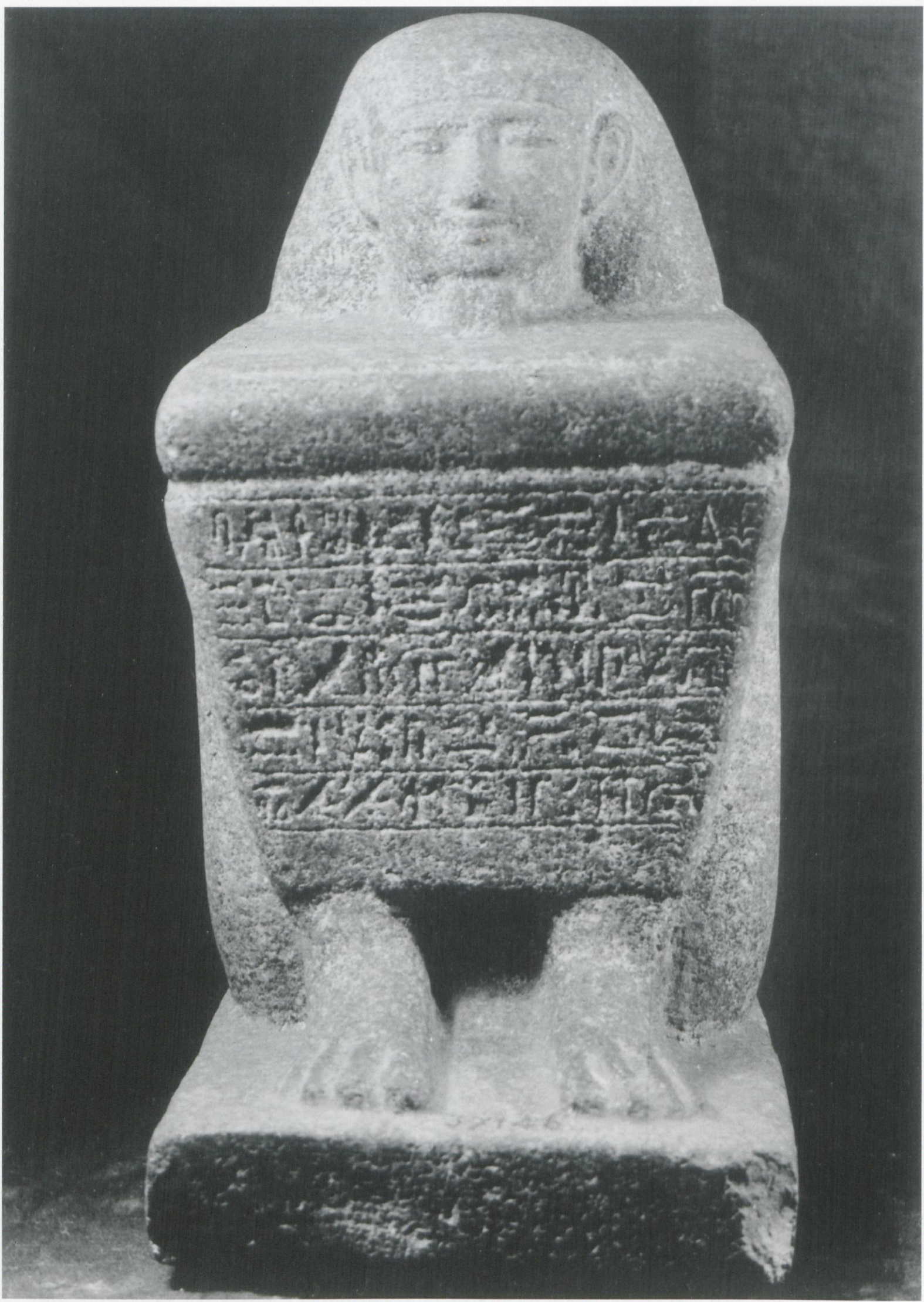

Würfelhocker Kairo JE 37146, Vorderseite (zu Jansen-Winkeln, Bau- und Bittinschrift). 


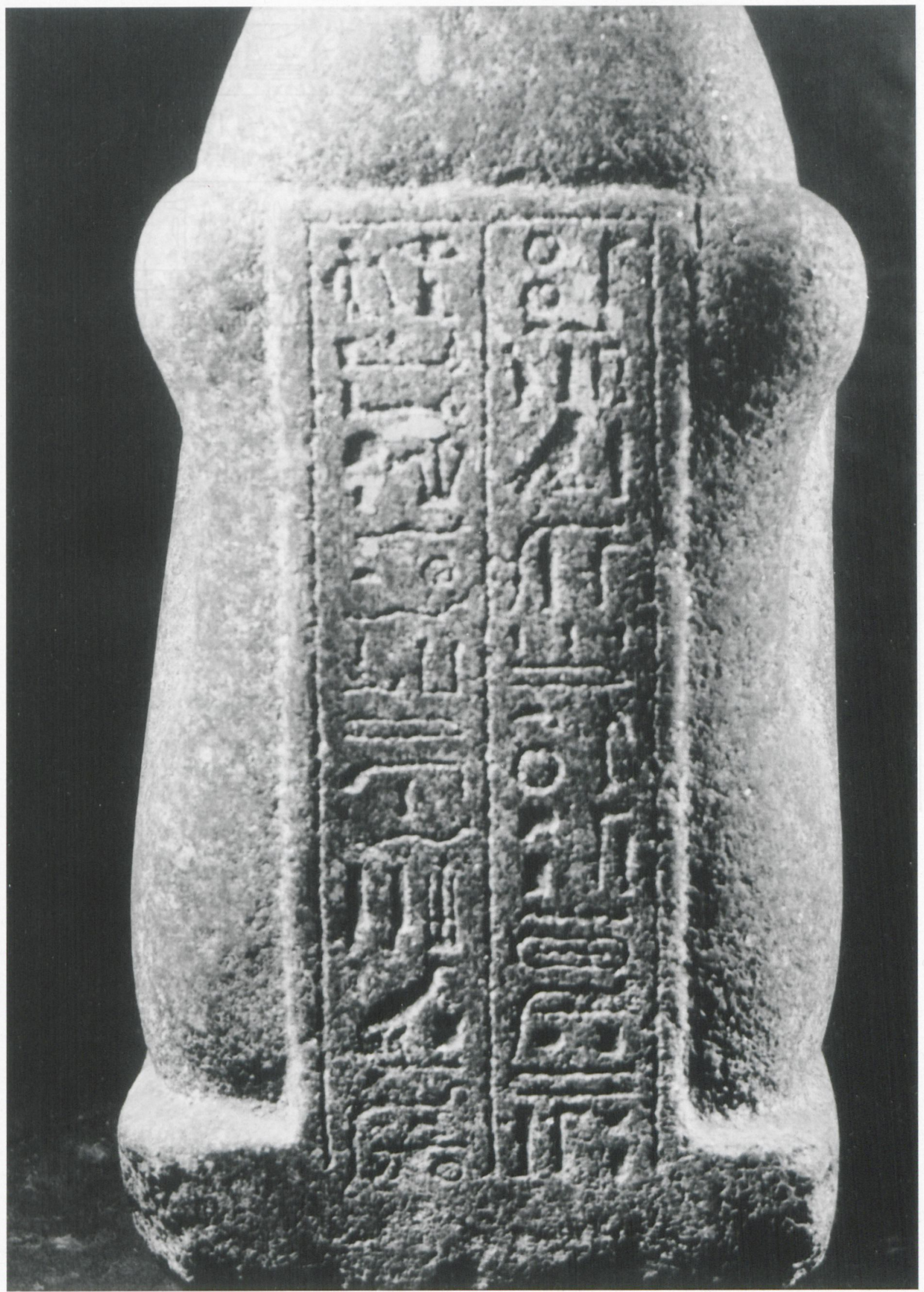

Würfelhocker Kairo JE 37146, Rückseite (zu Jansen-Winkeln, Bau- und Bittinschrift). 


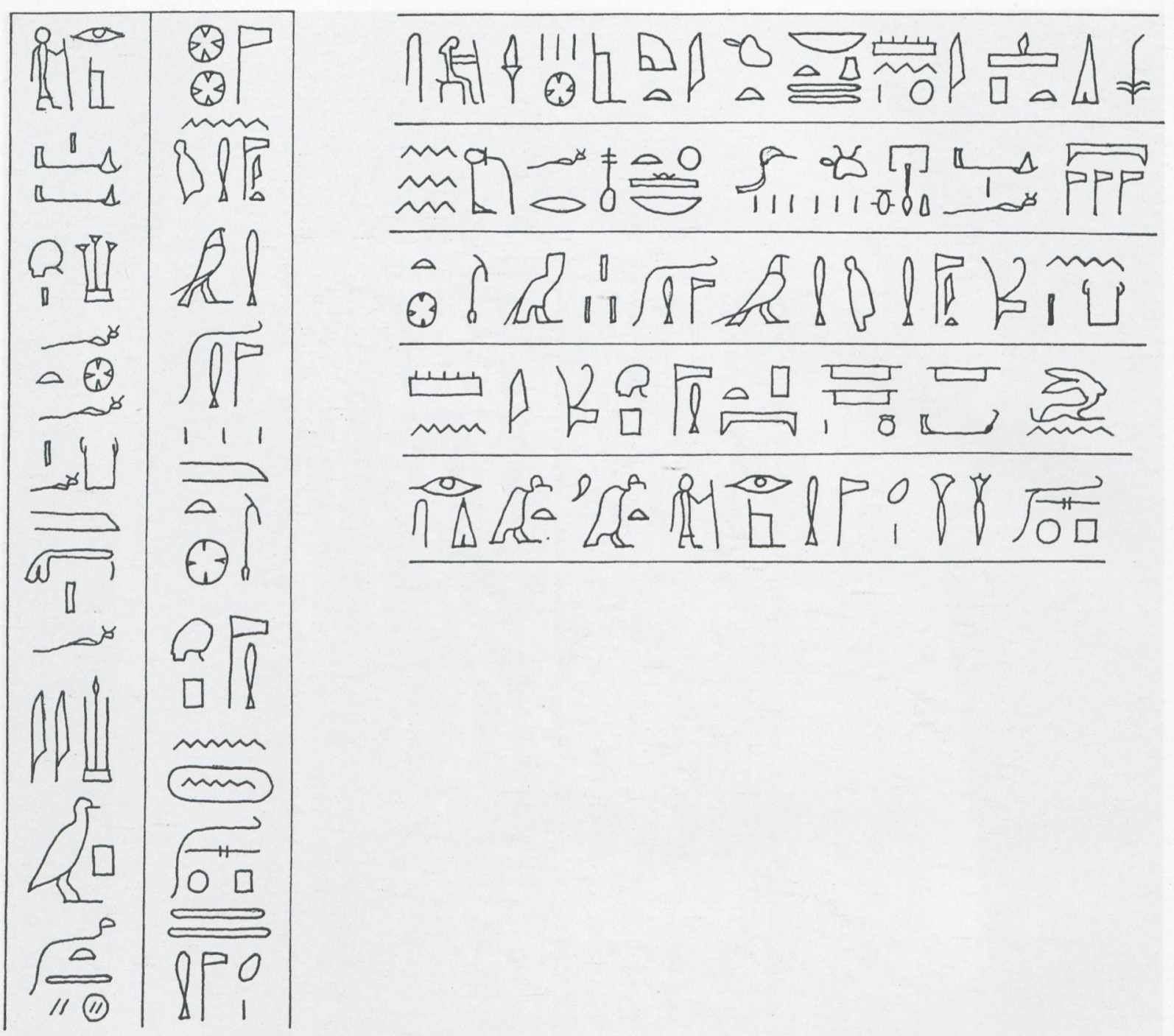

Text des Würfelhockers Kairo JE 37146, Vorderseite (rechts) und Rückseite (links)

(zu Jansen-Winkeln, Bau- und Bittinschrift). 